\title{
Adquisición de competencias profesionales en el Trabajo Fin de Máster: Colaborar para aportar soluciones reales
}

\author{
Irene Comeig Ramírez ${ }^{a}$ Alfredo Juan Grau Graub Lucía Pinar García ${ }^{c}$ Federico \\ Ramírez López y Amalia Rodrigo González ${ }^{\mathrm{e}}$ \\ a,b,d,e Departamento de Finanzas Empresariales, Facultat d’Economia (Universitat de València), Avda. \\ de los Naranjos, s/n, 46071 Valencia (Irene.Comeig@uv.es, Alfredo.Grau@uv.es, \\ Federico.Ramirez@uv.es y Amalia.Rodrigo@uv.es), 'Departamento de Economía Aplicada, Facultat \\ d’Economia (Universitat de València), Avda. de los Naranjos, s/n, 46071 Valencia \\ (Lucia.Pinar@uv.es)
}

\begin{abstract}
In order to motivate the student through a professional experience, and to improve the teaching-learning process by letting the student gain experience, we implement a collaborative learning-through-service methodology. This method increases the student involvement level and the deep understanding of the tools. As a matter of example, we present the implementation of this method in the Final Master Thesis (FMTh) of the Master on Corporate Finance at the University of Valencia (UV). In these FMTh, students have to valuate an actual innovation developed by UV researchers (on chemistry, in this case) with the goal of helping themto transfer (sell) this innovation to the industry. This collaborative learning-through-service methodology helps student put into practice the technics learned in the Master and give them a strong sense of the financial professional practice.
\end{abstract}

Keywords: Final Master Thesis, professional skills, collaborative work methodology, financial valuation.

\footnotetext{
Resumen

Con el objetivo de motivar al estudiante a través de una toma de contacto con la realidad profesional y mejorar el proceso de aprendizaje fomentando la interiorización de la técnicas de trabajo mediante un modelo colaborativo de tutorización, se plantea la inmersión del estudiante en un caso real al que debe aportar una solución. Aportamos como ejemplo la aplicación de esta técnica a la realización del Trabajo Fin de Master (TFM) del Master en Finanzas Corporativas de la Universidad de Valencia (UV). En estos TFMs, los estudiantes deben valorar un proyecto de inversión con consecuencias para la Transferencia de Resultados de Investigación de la UV: Se pretende responder a ¿Qué precio máximodebería estar dispuesta a pagar una empresa por los derechos de explotación de la investigación analizada? Para la realización de estos TFMs, se implementa la pedagogía Aprendizaje Servicio mediante la metodología de Trabajo Cooperativo que permite desarrollar una enseñanza centrada en la participación del alumnado favoreciendo el desarrollo de unos conocimientos más sólidos y duraderos.
} 
La innovación presentada permite al alumnado poner en práctica los conocimientos adquiridos en sus estudios de máster y les aproxima a la realidad empresarial..

Palabras clave:se trabajo fin de master, competencias profesionale, metodología de trabajo cooperativo, valoración financiera.

AGRADECIMIENTOS: Los autores agradecen el apoyo financiero del Vicerrectorado de Políticas de Formación y Calidad Educativa de la Universidad de Valencia, UV-SFPIE_RMD17-589360.

\section{Introducción}

$\mathrm{Al}$ final de cualquier master oficial los alumnos deben realizar su correspondiente Trabajo de Fin de Master (TFM), una práctica que cada vez es más común también en los títulos propios. La finalidad de este TFM es completar la asimilación de los conocimientos que se han adquirido durante el postgrado, desarrollando un ensayo teórico-práctico sobre algún aspecto relacionado con el master.

El RD 193/2007, de 29 de octubre, regula las enseñanzas universitarias oficiales y establece la obligatoriedad de completar el trámite del TFMpara conseguir el título de master oficial. Por otra parte, el RD 1027/2011, de 15 de julio, se centra en las competencias que deben adquirir los titulados en un master. Entre las competencias relacionadas con el TFM se incluyen, por ejemplo, el saber aplicar los conocimientos en entornos nuevos, evaluar y seleccionar la teoría científica adecuada y metodología precisas para formular juicios o saber transmitir claramente los resultados procedentes de la investigación científica.

Nuestra aportación a la innovación educativa radica en proporcionar al estudiante una opción colaborativa de inmersiónen la realidad profesional durante la realización del TFM. Con ello se mejora la motivación,y el proceso de aprendizaje-servicio fomenta la interiorizaciónde las técnicas profesionales que se adquieren mediante un modelo colaborativo de tutorización. En el ejemplo de aplicación que presentamos en la ejecución del TFM del Master en Finanzas Corporativas de la UV, el/la estudiante se pone en contacto con un grupo de investigación de la Universidad de Valencia (gracias a la publicidad hecha a través de los trípticos y de la página web desarrollada para dar publicidad de este proyecto de enseñanza-aprendizaje: RESEARCH+ CASHLab, y a contactos anteriores) y debe realizar como Trabajo de Fin de Máster (TFM) el análisis de la viabilidad financiera de la transferencia/venta a la industria de algún resultado de investigación de ese grupo. Concretamente, la pregunta que se pretende responder es: ¿Cuál es el precio máximo que una empresa debería estar dispuesta a pagar por laadquisición de los derechos de explotación de un producto de la investigación concreto generado por la Universidad de Valencia (patente, licencia, proceso, etc.)?

Este tipo de TFM ofrece un servicio de ayuda a la transferencia de resultados de investigación de las universidades, lo que motiva especialmente a los estudiantes y les traslada también la responsabilidad que sienten las/os encargados de las finanzas

(cc) BY-Nc-ND 2018, UniversitatPolitècnica de València

Congreso IN-RED (2018) 
empresariales. La valoración financiera del proyecto traduce a dinero y a rentabilidad la información sobre los productos derivados de la investigación, reduciendo así la asimetría de la información entre investigadores y empresarios compradores potenciales de dichos productos, lo que facilita la posterior transferencia de los resultados de la investigación al tejido empresarial.

\section{Objetivos}

El objetivo general que se plantea es que los estudiantes quefinalicen sus estudios de master sean capaces de desenvolverse en el cargo profesionalpara el que les prepara el máster. Para ello, se aplica una metodología de Trabajo Cooperativo para la adquisición de competencias profesionales.

Layer (2004) destaca que en estos úúltimos aaños las universidades manifiestan un destacado interés en la relación existente entre la Educación Superior y la inserción de sus titulados en el mundo laboral. Semeijnet al. (2005) advierte que todo esto le confiere al sistema universitario la gran responsabilidad a la hora de formar adecuadamente a su alumnado, para que en un futuro puedan desenvolverse de forma eficiente en sus puestos de trabajo.

Las competencias educativas, por un lado, robustecen las posibilidades de lograr un trabajo que no tiene por quéestar estrechamente asociado al área formativa, por otro lado, refuerzan las posibilidades de que puedan desarrollar de forma óptima las exigencias del puesto a ocupar (Heijkeet al., 2003).

Las competencias que se definen tienen que ser evaluables y por ello, la forma en la que éstas se formulen debe permitir la identificación de resultados de aprendizaje que puedan ser observables y mensurables (Riesco, 2008).

La Tabla 1 presentacomo ejemplo las competencias básicas y que se pretende desarrollar en los alumnos a través de la experiencia que implementamos.

La experiencia de innovación educativa que presentamos, utiliza la metodología de Trabajo Cooperativoy, concretamente, se desarrolla la pedagogía del Aprendizaje Servicio (APS) en los alumnos del master. Puig et al. (2009) definen de diferentes formas el APS incidiendo en dos componentes básicos: a) la eficacia del aprendizaje y la aplicación curricular de procedimientos y actitudes, b) el servicio volcado a la comunidad en este caso con la transferencia de resultados de investigación. Siguiendo a Puig et al (2006), el APS quiere dar respuestas a necesidades reales de la sociedad impulsando la participación y cooperación de los estudiantes con otras instituciones y entidades. Se impulsa, por tanto, con el APS la educación en valores a través de su práctica, permitiendo que los estudiantes puedan usar los conocimientos adquiridos en el master como una herramienta que les permita aportar soluciones reales al tejido socio-empresarial. 
Tabla 1. Competencias profesionales

\begin{tabular}{|c|c|}
\hline Código & Descripción \\
\hline CB2 & $\begin{array}{l}\text { Que los estudiantes sepan aplicar sus conocimientos a su trabajo o vocación de una } \\
\text { forma profesional y posean las competencias que suelen demostrarse por medio de la } \\
\text { elaboración y defensa de argumentos y la resolución de problemas dentro de su área } \\
\text { de estudio }\end{array}$ \\
\hline CG6 & Habilidad para analizar y buscar información proveniente de fuentes diversas \\
\hline CG12 & Capacidad para trabajar en equipo \\
\hline CG17 & Capacidad de aprendizaje autónomo \\
\hline CE2 & $\begin{array}{l}\text { Ser capaz de elaborar e interpretar la información contable, tanto la financiera, } \\
\text { destinada a los usuarios externos, como la interna, destinada al control de gestión y la } \\
\text { toma de decisiones }\end{array}$ \\
\hline CE4 & $\begin{array}{l}\text { Ser capaz de resolver problemas de valoración financiera, tanto en decisiones de } \\
\text { financiación, como de inversión empresarial, en el entorno nacional e internacional }\end{array}$ \\
\hline CE8 & $\begin{array}{l}\text { Conocer y analizar los mercados financieros, así como las operaciones financieras } \\
\text { anejas al ámbito empresarial }\end{array}$ \\
\hline CE49 & $\begin{array}{l}\text { Conocer los fundamentos básicos que rigen las operaciones y los mercados } \\
\text { financieros }\end{array}$ \\
\hline CE52 & $\begin{array}{l}\text { Capacidad para medir y valorar el riesgo de los activos financieros y sus aplicaciones } \\
\text { en las inversiones productivas }\end{array}$ \\
\hline CE56 & $\begin{array}{l}\text { Conocer los diferentes instrumentos de financiación y ser capaz de determinar la } \\
\text { política de endeudamiento de la empresa }\end{array}$ \\
\hline
\end{tabular}

Fuente: www.uv.es/economia (Grado en Administración y Dirección de Empresas)

Concretamente, con nuestra aportación a la innovación educativa de inmersión en la realidad profesional durante la realización del TFMse pretende reducir la brecha entre formación académica y el trabajo a desempeñar en la empresa donde se desarrollen laboralmente. Esta aportación:

1) Proporciona una primera toma de contacto al estudiante demaster con el díaa adía real de un profesional.

2) Mejora el proceso de enseñanza-aprendizaje fomentando la interiorización de la técnicas de trabajo del estudiante mediante la inmersión del estudiante en un caso real con un cliente real y consecuencias (para la Transferencia de Resultados de Investigación a la industria).

3) Fomenta la participación del estudiante realizando un aprendizaje-servicio, mediante una técnica de trabajo en colaboración con el grupo de investigación y con la/el profesor/a tutor/a. 
4) Pone en contacto el estudio universitario con el mercado laboral, puesto que el estudiante podrá participar en las entrevistas con las empresas interesadas en comprar la investigación, en nuestra aplicación concreta.

5) Impacta positivamente no solo en la formación del estudiante sino en la transferencia de resultados de investigaciónn de la Universidad de Valencia al tejido industrial.

6) Dado que los trabajos de Fin de Master están demostrando ser hasta ahora el talón de Aquiles de los masters profesionales, esta innovación educativa representa una posible solución para cambiar la tendencia.

\section{Desarrollo de la innovación}

\subsection{Metodología de Trabajo Cooperativocon Aprendizaje Servicio}

Para desarrollar la innovación planteada se ha seleccionado la metodología de Trabajo Cooperativo. La principal ventaja de este método es que permite desarrollar una enseñanza centrada en la participación del alumnado donde la responsabilidad del aprendizaje depende estrechamente de su nivel de implicación y compromiso. Ello conduce a adquirir unos conocimientos con mayor profundidad, interiorizarlos y, en consecuencia, favorecen su durabilidad a lo largo el tiempo y su aplicabilidad a distintos campos y situaciones.

Esta metodología se caracteriza por fomentar la adquisición de conocimientos, habilidades o actitudes como resultado del trabajo en grupo o, en otras palabras, un aprendizaje individual como resultado de un proceso grupal (Ariza, 2000).

Además, la participación e implicación personal del estudiante en el desarrollo del proceso para aportar una solución a un problema real interioriza el aprendizaje y fija los conocimientos con carácter duradero (Ramírez y Grau, 2016; Comeig y JaramilloGutiérrez,2011)

En la experiencia que implementamos de Aprendizaje Servicio (APS), la relación de cooperación no se limita a un contacto bidireccional entre docente y estudiante, sino que el alumno cuenta con, además del apoyo académico y científico del profesor, los conocimientos del grupo para el que realiza el trabajo (“cliente”). De esta forma, el resultado final se beneficia de las sinergias que implica trabajar con académicos(docente) y prácticos ('cliente').

El docente asume la figura de mediador entre los distintos integrantes del grupo, así como de garante de una actividad que se realice de forma satisfactoria para todas las partes implicadas. Se le confiere también la responsabilidad de promover la capacidad de reflexión en sentido constructivo ycrítico.

En nuestro ejemplo, el/la estudiante se pone en contacto con un grupo de investigación de la Universidad de Valencia (gracias a la publicidad hecha a través de los trípticos y de la página web desarrollada para dar publicidad de este proyecto de enseñanza- aprendizaje, RESEARCH+CASHLab, principalmente) y debe realizar como TFM el análisis de la 
viabilidad financiera de la transferencia/venta a la industria de algún resultado de investigación de ese grupo.

Este tipo de TFM motiva sustancialmente a los estudiantes y también les traslada la responsabilidad que sienten las/os encargados de las finanzas empresariales. La valoración financiera del proyecto reduce la asimetría de la información entre investigadores y empresarios compradores potenciales de dichos productos, lo que facilita la posterior transferencia de los resultados de la investigación al tejido empresarial.

Para analizar este proyecto de inversión se utilizan los indicadores financieros de valoración tradicionales: Cálculo de los flujos netos de caja del proyecto, VAN, TIR, análisis de sensibilidad y punto muerto, etc

\subsection{Ejemplo de aplicación de la opción colaborativa de inmersión en la realidad profesional durante la realización del TFM}

La innovación mediante elAprendizaje Servicio de los alumnos del Master de Finanzas Corporativas se integra con la metodología de Trabajo Cooperativopor la colaboración entre distintosgrupos de investigación y docencia. En nuestro ejemplo el grupo MINTOTA del Departamento de Química Analítica (Facultad de Química-UV) tiene una investigación que le interesaría llevar al mercado empresarial, el grupo RESEARCH+CASHLab del Departamento de Finanzas Empresariales (Facultad de Economía-UV) tiene los conocimientos para dar una valoración económico-financiera a ese resultado de investigación, y el/la estudiante del Máster en Finanzas Corporativas tiene el deseo de fijar los conocimientos en Finanzas Empresariales al realizar su TFM.

Como ejemplo de nuestra metodología de innovación educativa, presentamos un TFM dedicado a la valoración de un producto de la investigación del grupo MINTOTA del Departamento de Química Analítica (Facultad de Química-UV), que consiste en un sensor colorimétrico in situ que detecta la cantidad de aminas presentes en el aire.Estos sensores pueden ser de gran utilidad en la industria de la alimentación, ya que, al indicar el nivel de contaminantes (aminas) del alimento que incorpora este dispositivo, en función del color que muestra, permite identificar su grado de salubridad previo a ser consumido.

El RESEARCH+CASHLab, formado por investigadores del Departamento de Finanzas Empresariales (UV), representa el punto de encuentro entre investigadores, inversores y tejido empresarial. Por ello, está especialmente bien posicionado para ofrecer apoyo académico y científico a aquellos alumnos del Master en Finanzas Corporativas que soliciten desarrollar su trabajo fin de master bajo esta metodología de aprendizaje servicio. Se dedican a valorar innovación científica (ej. nuevos hallazgos relevantes, patentes, licencias) para que puedan llegar al mundo de la empresa y a la sociedad.

Las fases en que se desarrolla la valoración de los dispositivos pasivos son:

\section{FASE I. REUNIÓN CON EL GRUPO DE INVESTIGACIÓN}

En primer lugar, se reúnen el alumno del master de Finanzas Corporativas y su profesortutor con los miembros del grupo de investigación, en este caso MINTOTA. De esta primera reunión se extrae información relevante sobre las características del dispositivo 
pasivo atmosférico y sus ventajas diferenciadores frente a sus principales competidores. Se le proporciona todo tipo de datos inherentes al dispositivo y que serán necesarios para hacer la posterior valoración financiera.

\section{Fase II. Diseño de las hipótesis de Trabajo y los Estados Financieros Previsionales}

Con toda la información recabada, el alumno junto con el profesor-tutor y el apoyo adicional de RESEARCH+CASHLab diseñan las hipótesis de trabajo que servirán para estimar todas las magnitudes que concurren en el cálculo de la rentabilidad en la explotación de estos sensores pasivos.

Las hipótesis que se plantean son:

- Hipótesis sobre la estimación de los costes directos laborales: personal a jornada parcial, número de sensores a fabricar por hora, etc.

- Hipótesis sobre la estimación del crecimiento de las ventas.

- Hipótesis sobre la estimación de los costes fijos. publicidad, amortización derivado de la obtención de la licencia, etc.

- Hipótesis sobre la estimación de la Cuenta de Pérdidas y Ganancias.

\section{FASE III. CÁlCUlo de los FluJos Netos de CAJA}

Una vez establecidas las hipótesis de partida, el alumno estima los Flujos Netos de Caja (FNC) derivados de la realización del proyecto y necesarios para analizar los distintos instrumentos financieros conducentes a decidir si el proyecto será rentable.

\section{FASE IV. ESTUDIO Y ANÁLISIS DE LOS INSTRUMENTOS FINANCIEROS}

Seguidamente, el alumno hace uso de los distintos métodos de valoración a efectos de analizar la viabilidad financiera del proyecto planteado. Estos son:

- Valor Actual Neto (VAN). Medida de la rentabilidad absoluta neta. Este indicador va a servir para identificar si el valor del proyecto es superior a su coste, tomando en consideración la tasa del coste de oportunidad del capital invertido.

- Tasa Interna de Rentabilidad (TIR). Medida de la rentabilidad en términos relativos brutos y permite, al igual que el VAN, decidir si acometer o no el proyecto.

- Umbral de Rentabilidad Financiero o Punto Muerto (PM). Nos indica cuantos sensores hay que producir para igualar el valor con el coste y, conocer así, cuántas unidades se han que vender para empezar a obtener un VAN positivo.

- Análisis de Sensibilidad (AS). Con el fin de analizar posibles riesgos derivados de desviaciones en la estimación de las hipótesis iniciales en cuanto a ventas o costes adicionales, se plantea un análisis de sensibilidad. Esto permite identificar aquellos escenarios en los que el proyecto podría fracasar y, así, llevar un mayor control de estas contingencias.

- Método de Montecarlo (MM). Este método introduce aleatoriedad en una serie de variables definidas por el usuario para estudiar el comportamiento del modelo 
planteado. En este caso, se han establecido unos valores mínimos y máximos que ciertas variables podrían tomar y se han generado 1.000 valores diferentes del VAN según los diferentes valores de las variables aleatorias consideradas.

\section{FASE V. EMISIÓN DEL INFORME EJECUTIVO FINAL}

Finalmente, el alumno emite dos informes ejecutivos. El primero con todo el desarrollo al completo y el segundo más abreviado. Estos documentos se le entregan al grupo de investigación, en este ejemplo, MINTOTA, para corroborar la viabilidad financiera de la explotación de los dispositivos pasivos y, así, plantearse la explotación de su licencia.

Por otro lado, el alumno redacta su trabajo fin de master que recogerá todos los desarrollos mencionados anteriormente y que se ajustan a la guía académica del mismo.

\section{Resultados}

Como consecuencia de la aplicación de metodología colaborativa para aportar soluciones reales (aprendizaje servicio) en la realización del TFM, se adquieren competencias profesionales duraderas. El/la estudiante interioriza las cuestiones tratadas (en nuestro ejemplo: la valoración financiera tratada durante el Máster). Además, seadquieren competencias prácticas no solo sobre valoración financiera, sino también sobre trabajo en equipo, trasmisión de ideas, recopilación de información, gestión del tiempo y de la responsabilidad, etc. En definitiva, el/la estudiante adquiere experiencia profesional (en nuestro ejemplo sobre las finanzas empresariales), y de la gestión del trabajo en equipo, pero siempre bajo la tutela y con la colaboración del profesor/a.

En nuestro ejemplo, los resultados obtenidos con esta experiencia de innovación son de dos tipos: el académico y el de transferencia de investigación. En primer lugar, desde el punto de vista académico: (i) gracias a la elaboración del trabajo fin de master con la valoración de la explotación de la licencia del dispositivo pasivo para la detección in situ de aminas en gases, el alumno además de poder aplicar los conocimientos teóricos adquiridos, entra en contacto con empresas reales valorando proyectos de inversión en un contexto real; y (ii) en muchas ocasiones estos estudiantes que han desarrollado estas tareas de valoración, han acabado trabajando en las empresas donde han desempeñado esta actividad profesional.

Por otra parte, en el ámbito de la transferencia de resultados de investigación, (i) al grupo MINTOTA se le facilita una serie de informes ejecutivos de viabilidad económicofinanciera que le permitirán comercializar su licencia; y (ii) el grupo RESEARCH+CASHLab, está contribuyendo a la transferencia de los resultados de investigación a la empresa dado que facilita informes que permiten a los investigadores vender los resultados de su producción científica.

\section{Conclusiones}

La aplicación de metodología colaborativa para aportar una solución a un problema real (aprendizaje servicio) en la realización del TFM, ha permitido adquirir competencias 
profesionales duraderas. El/la estudiante interioriza los conocimientosimplicados en la solución. Además, seadquierenotras competencias prácticas como: trabajo en equipo, comunicación, recopilación de información significativa, y gestión del tiempo y de la responsabilidad. En definitiva, el/la estudiante adquiere experiencia profesional pero bajo la tutela y con la colaboración del profesor/a.

Llevar a cabo esta experiencia de innovación ha permitido la toma de contacto con el mundo real empresarial por parte del alumnado, y le ha permitido desarrollar gran parte de las competencias profesionales que precisan para desempeñar adecuadamente un puesto de trabajo en el área financiera.

Una de las principales ventajas de la colaboración interdepartamental (en nuestro ejemplo: MINTOTA, RESEARCH+CASHLab y Máster en Finanzas Corporativas), es que permite desarrollar con una mayor calidad los trabajos fin de máster en Finanzas Corporativas con una mayor motivación e interiorización por parte de los estudiantes, en un contexto real empresarial. Se ha comprobado que se han producido efectos sinérgicos que han favorecido a los resultados obtenidos tanto en el ámbitodocente-académico como en el científico.

Trabajar en esta línea de canalización del aprendizaje a ofrecer un servicio mediante la colaboración, da resultados muy positivos. En nuestro ejemplo, gracias al apoyo académico y científico prestado por RESEARCH+CASHLab. De esta forma se logra aproximar los intereses de los distintos grupos involucrados: alumnos, docencia, investigadores y empresas.

\section{Referencias}

ARIZA, A. (2000) "Las Nuevas Tecnologías de la Información y la Comunicación y una Propuesta para el Trabajo Colaborativo" en V Congreso Iberoamericano de Informática Educativa. http://www.edudistan.com/Adolfo\%20Ariza.htm (consultado el 10 de noviembre de 2017)

COMEIG, I.. y JARAMILLO-GUTIÉRREZ, A. (2011) "Experimentosinteractivospara la enseñanza de economía y finanzas: unaaplicación a la docencia de estadística” en Experiencias de innovacióndocente en estadistica, Universidad de Valencia, Valencia. p. 191-199.

España. Real Decreto 1393/2007, de 29 de octubre, por el que se establece la ordenación de las enseñanzas universitarias oficiales (publicado en BOE núm. 260, de 30 de octubre de 2007). Disponible en: https://www.boe.es/diario boe/txt.php?id=BOE-A-2007-20202 (consultado el 20 de diciembre de 2017).

España. Real Decreto 1027/2011, de 15 de julio, por el que se establece el Marco Español de Cualificaciones para la Educación Superior (publicado en BOE núm. 185, de 3 de agosto de 2011). Disponible en: https://www.boe.es/buscar/doc.php?id=BOE-A-2011-13317 (consulta el 20 de diciembre de 2017). 
HEIJKE, H., MENG, C. y RIS, C. (2003). "Fitting to the Job: The Role of Generic and Vocational Competencies in Adjustment and Performance" en Labour Economics, vol. 10, p. $215-229$

LAYER, G. (2004). Widening Participation and Employability. York: Learning and Teaching Support Network.

MUÑOZ, F., ARVAYO, K., VILlEGAs, C., GONZÁLEZ, F. y SOSA, O. (2014). “El método colaborativo como una alternativa en el trabajo experimental de Química Orgánica” en Educación Química, vol. 25, issue 4, p.464-469.

PUIG, J.M. y PALOS,J. (2006). "Rasgos pedagógicos del aprendizaje-servicio” en Cuadernos de Pedagogía, vol. 357, p.60-63.

PUIG, J.M., GIJON, M., MARTÍN, X. y RUBIO. L. (2009). Aprendizaje Servicio. Educación y compromiso cívico. Barcelona: Graó.

RAMÍREZ, F. y GRAU, A. J. (2016). “La Innovación Educativa y la Economía Experimental: Un experimento sobre el contagio del pánico bancario” en Innovación Universitaria: Digitalización 2.0 y Excelencia en Contenidos. Mc Grau Hill/ Interamericana. Madrid. p. 665-676.

RIESCO, M. (2008). "El enfoque por competencias en el EEES y sus implicaciones en la enseñanza y el aprendizaje” en Tendencias pedagógicas, vol. 13, p. 79-106.

SÁEZ, J. y RUÍZ, J.M. (2012). "Metodología didáctica y tecnología educativa en el desarrollo de las competencias cognitivas: aplicación en contextos universitarios” en Revista de Currículum y Formación del Profesorado, vol. 16, issue 3, p. 373-391.

SEMEIJN, J.H., VELDEN, R.K.., HEIJKE, J.A.., VLEUTEN, C.P. y BOSHUIZEN, H.C. (2005). Competence Indicators in Academic Education and Early Labour Market Success of Graduates in Health Sciences. Maastricht: Research Centre for Education and the Labour Market. 